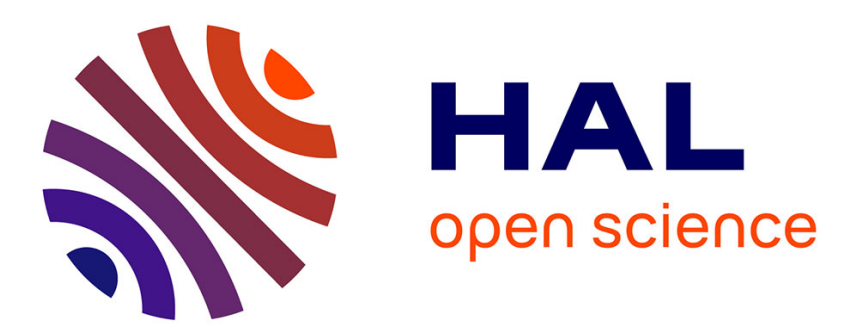

\title{
Vacuum Casting to Manufacture a Plastic Biochip for Highly Parallel Cell Transfection
}

Matthieu Denoual, Y. Macé, Bruno Le Pioufle, Pascal Mognol, D. Castel, X. Gidrol

\section{- To cite this version:}

Matthieu Denoual, Y. Macé, Bruno Le Pioufle, Pascal Mognol, D. Castel, et al.. Vacuum Casting to Manufacture a Plastic Biochip for Highly Parallel Cell Transfection. Measurement Science and Technology, 2006, 17, pp.3134-3140. 10.1088/0957-0233/17/12/S03 . hal-00739220

\section{HAL Id: hal-00739220 https://hal.science/hal-00739220}

Submitted on 20 May 2014

HAL is a multi-disciplinary open access archive for the deposit and dissemination of scientific research documents, whether they are published or not. The documents may come from teaching and research institutions in France or abroad, or from public or private research centers.
L'archive ouverte pluridisciplinaire HAL, est destinée au dépôt et à la diffusion de documents scientifiques de niveau recherche, publiés ou non, émanant des établissements d'enseignement et de recherche français ou étrangers, des laboratoires publics ou privés. 


\title{
Vacuum Casting to Manufacture a Plastic Biochip for Highly Parallel Cell Transfection
}

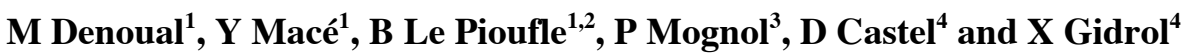 \\ ${ }^{1}$ CNRS BIOMIS/SATIE, ENS de Cachan, campus de Ker-Lann, 35170 Bruz, France \\ ${ }^{2}$ CNRS LIMMS, University of Tokyo, Komaba 4-6-1, Meguro-Ku, Tokyo 153-8505, Japan \\ ${ }^{3}$ CNRS IRCCYN, 1 rue de la Noë, BP 92101, 44321 Nantes Cedex 03, France \\ ${ }^{4}$ CEA, Service de Génomique Fonctionnelle, 2 rue G. Crémieux, 91057 Evry, France
}

Email :denoual@bretagne.ens-cachan.fr

\begin{abstract}
A novel polymer microarrays fabrication technique is presented, and applied to the realization of a new type of biochip for cellulomics purposes. The proposed microfabrication technique is optimized for production cost reduction when small series (100 to 10000 chip series) are needed, which is the case when the actual market of cellulomic is considered. As a demonstration, a biochip for highly parallelized transfection of arrays of living cells was realized, and the feasibility of parallel lipofection on this biochip was demonstrated. Two different plasmids encoding respectively eGFP and DsRED2 were inserted into HEK293T cells. The transfection was monitored through fluorescence observation after 72 hours. Successful expression of both genes was observed.

The proposed microfabrication technique is derivated from a macroscale rapid prototyping technique called vacuum casting. The master part is here performed through combining microfabrication technologies and rapid prototyping technologies. The corresponding threedimensional female structure, combining micro and macro-size features is molded in a flexible silicone-material. The duplicated polymer chips are obtained by casting a thermosetting plastic under vacuum. The dimensional replication accuracy between the master part and the duplicated parts is uniform over the duplicated parts and better than $1 \%$. Advantages of the proposed technique compared to existing plastic microfabrication techniques are discussed in the paper.
\end{abstract}

Keywords: Cell array, vacuum casting, transfection, Microsystem, plastic biochips, polymer technology, DNA

PACS numbers: 47.54.Jk, 47.57.Ng, 47.54.Fj

\section{Introduction}

Micromachining and Micro-Electro-Mechanical-Systems (MEMS) technology have been advantageously used in biological and biomedical-related fields since last decade. Indeed the 
miniaturization opened new perspectives to those fields thanks to the new possibilities to handle or analyze biocomponents, with scale laying from the dimensions of a living cell down to the dimensions of biomolecules [1-6].

One of the miniaturization interest is the integration on a single chip of a large number of tests or biological reactions. This concept of parallelization is already widely used in the context of DNAchips or protein chips $[7,8]$. This approach is nowadays extending to parallel analysis of living cells monitoring with the recently introduced concept of parallel cellchips $[9,10]$, for high throughput screening (HTS) that find their applications in the fields of pharmaco-toxicology, cosmetology or food industry for example. Parallel cellchips are constituted of a large number of microwells in which cells are spotted and exposed to biochemical products which efficiency or toxicity has to be evaluated.

Historically, microsystems for biological applications have been developed on silicon, glass or fusedsilica [11-15]. The choice for silicon as material was motivated by technological facilities available in the silicon microfabrication industry, while the choice for glass or fused-silica was guided by the advantages of providing a transparent support for biological analysis. Indeed an important drawback of silicon chips, as far as biological or optical use is concerned, is that this material does not fulfill the requirement of transparency. In the other hand, the glass micromachining is not as convenient as silicon micromachining, and this technology remains expensive. That is why, because of cost, material properties, or availability of micromachining methods, the microtechnologies used to design biochips (as well as optical microsystems or Radio Frequency micro electromechanical systems RFMEMS [16] progressively turned to polymer casting since the last decade [17].

Several types of polymer micro-fabrication techniques have been demonstrated. Among them, two categories can be pointed out: 1) micro-fabrication technologies which are derived from a macro-scale polymer technologies: for instance injection molding led to micro-injection molding, while hot embossing led to micro hot embossing 2) technologies which are derived from clean-room microtechnologies: the PDMS (PolyDi-MethylSiloxane) soft-lithography [18], or the recently proposed UVembossing techniques are representative examples of that category $[19,20]$.

Micro-technologies of the first category [21-25] require huge investments in terms of money, equipments and time. They need high pressure and temperature control in order to cast the thermoplastic materials which are used. In order to miniaturize the three dimensional mould, its fabrication needs technology more precise than the one encountered with conventional computer numerical control (CNC) machining or micromachining (which is nowadays limited down to $40 \mu \mathrm{m}$ dimensions), and is thus turning to microtechnologies such as LIGA (Lithographie Galvanoformung Abformung), or deep silicon etching. Indeed latter micromachining methods permit to achieve submicron resolution for the mold. However those techniques cannot cope with the non-uniform retraction phenomena of the polymer material duplicated parts. The studies show that the deformation of the polymer parts depends on local mould temperature, injection point, and of the moulded objects volume and geometry $[26,27]$. This limitation is strongly unfavourable for highly parallel devices, as 
microarrays, as in this case a non uniform retraction leads to the loss of dimensional periodicity. Indeed periodicity is a key feature for microarrays spotting and high data volume acquisition automation.

This limitation explains the recent increase of interest on UV-lithography based technologies [28] which have the advantage to ensure uniform periodicity of micropatterns and highly accurate replication. Those technologies are low temperature based on pattern transfert from a microtechnology fabricated mould part to a UV curable resin. Nevertheless, the range of possible materials is limited to UV-curable resin and does not allow a wide range of mechanical, optical properties and the biocompatibility of each material should be carefully studied. Moreover, the cost of such material is quite high compared to thermoplastic material cost.

PDMS soft-lithography [18] is a widely known technology that can be used for microdevices prototyping or in some cases as complete devices [29]. Nevertheless PDMS material properties are not convenient for microarrays fabrication. Indeed the low stiffness of PDMS renders it difficult to manipulate without additional support. Moreover biological results are hardly reproducible on PDMS devices because of prepolymer partial polymerisation, which leads to PDMS porosity to surrounding medium molecules.

In this paper, we present an alternative low temperature polymer micro-duplication technology that allows the serial fabrication of polymer micro-devices suitable for biological and microfluidic application. The proposed technique derives from a macroscale rapid prototyping fabrication technique called vacuum casting. This technology is based on the duplication of a master part through a female flexible mould into a thermosetting polymer by the mean of casting under vacuum. Using this technique one can fabricate several hundreds micro-devices with dimensions and aspect-ratios suitable for biomicrosystems applications. Moreover, the thermosetting polymers involved in this technique show excellent biocompatibility and fluidic properties and reveal excellent properties for fluorescence observation. Microarrays have been performed using this technology and used for DNA transfection experiments on cells. Those experiments were carried out on HEK293T cells. The transfection of plasmid encoding eGFP and DsRED2 was monitored through fluorescence observation. The next section presents the fabrication technology and the microarray design, while last section of the paper describes the cell on a chip transfection experiments.

\section{Fabrication and design}

In this paragraph the proposed microfabrication method, allying polymer vacuum casting to microsystem technology, is presented. As we shall see the obtained products combines advantages of both technologies, mainly low cost production, capability to reach small size features, and low retraction after molding. The fabrication of the cell microarray plastic biochip is then described . 


\subsection{Vacuum casting of microsized structures process}

The proposed micro-duplication technology derives from a macroscale vacuum casting technique that follows three steps. The first step consist in the realization of a master part that corresponds in dimensions and geometry to the final desired polymer part. Then a flexible silicone mould is hardened surrounding the master part so as to transfert the micro and macro patterns to a female flexible counterpart. The use of silicone material allows the use of various techniques for the fabrication of the master part. Silicon masters that are too fragile for high pressure processes are directly usable in that case, without any reinforcement. Lastly, thermosetting polymer is casted under vacuum into the flexible mould and hardens until it is withdrawn from the flexible mould as a duplicated part.

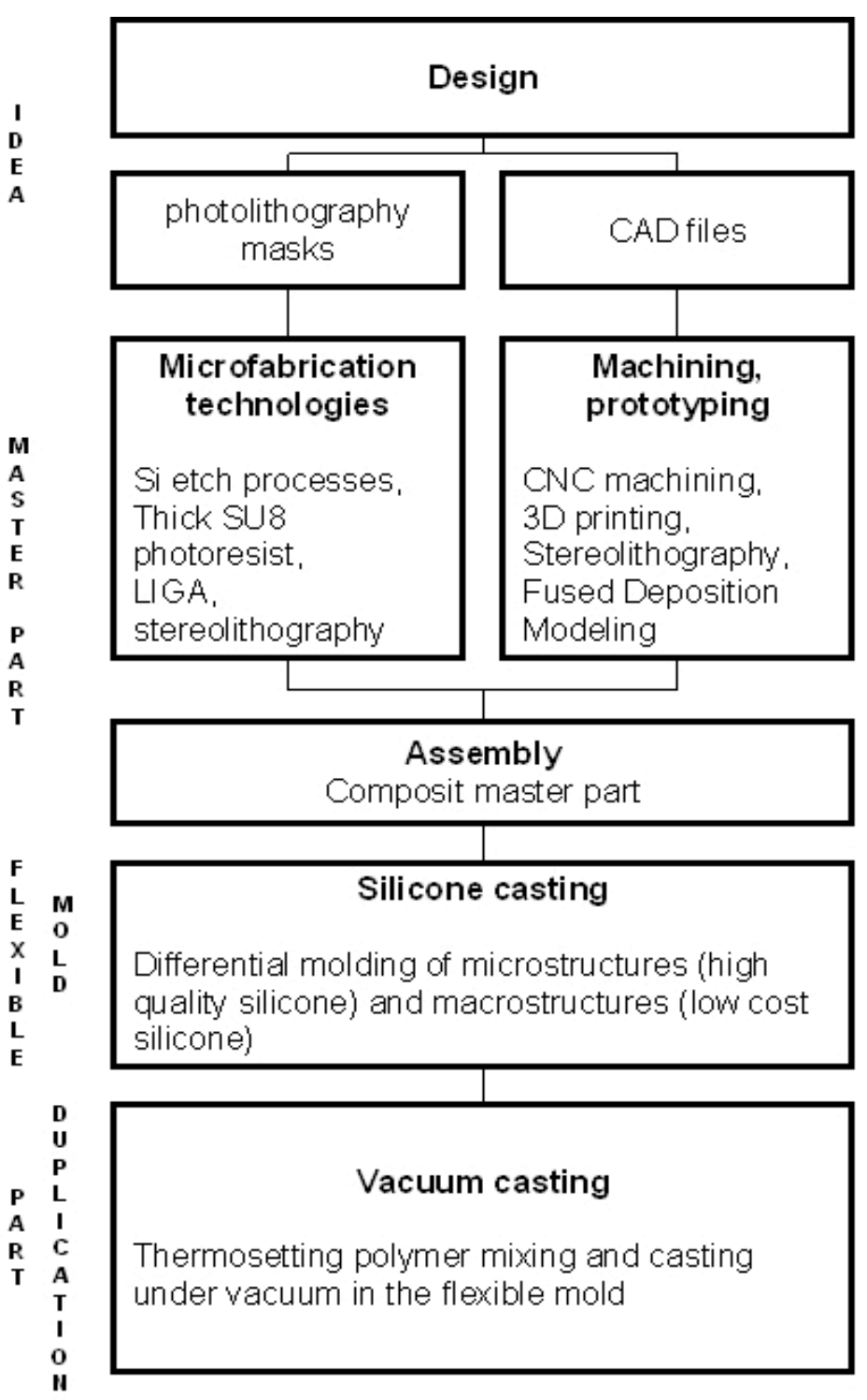

Figure 1: General process flow for vacuum casting technique of polymer micro-sized chips. 
The performances of this polymer micro-fabrication technique have been evaluated through its replication capabilities of micron-scale, high-aspect ratio and submicron scale structures [30]. The duplications of classical tests structures showed sub-micrometer (down to $300 \mathrm{~nm}$ ) and high aspectratio (up to 1:16) performances. The results highlight that vacuum casting fits the requirements for a prototyping technique allowing the development and fabrication of polymer microsystems. This technology allows highly accurate duplication of microstructure with uniform contraction of maximum $1 \%$. The uniform contraction observed could be explained by two major characteristics of this technology that differentiate it from existing duplication techniques. Firstly, it is a low temperature (below $80^{\circ} \mathrm{C}$ ) and low pressure process using liquid mixture of thermosetting polymers, therefore stresses to polymer material are much lower than in high temperature and high pressure processes cases. Secondly, the mould use for the replication of the microstructure is flexible, which means that during the cooling down, after the thermosetting polymer is hardened, no external stress is applied to the polymer material as it is the case during the cooling down of polymer material in stiff metal molds. This performance ensure the highly accurate patterns periodicity necessary for microarray applications.

\subsection{Cell Microarray design}

The microfabricated cellchip array consists of a matrix of 2025 pyramidal microwells. The dimensions of the bottom of microwells $(200 \mu \mathrm{m} \times 200 \mu \mathrm{m})$ allow the culture of an average number of 30 to 50 cells, which is sufficient to keep a good biological viability and functionality for the type of adherent cells chosen here. The depth of the wells is $50 \mu \mathrm{m}$ to ensure enough medium volume for cell culture. The smallest dimension on the chip is $30 \mu \mathrm{m}$, corresponding to the microwells separation, and the maximum aspect ratio is $1: 3$. The microwells matrix takes place in a global package fitting the usual biological glass slide features for simple manipulation.

(a)

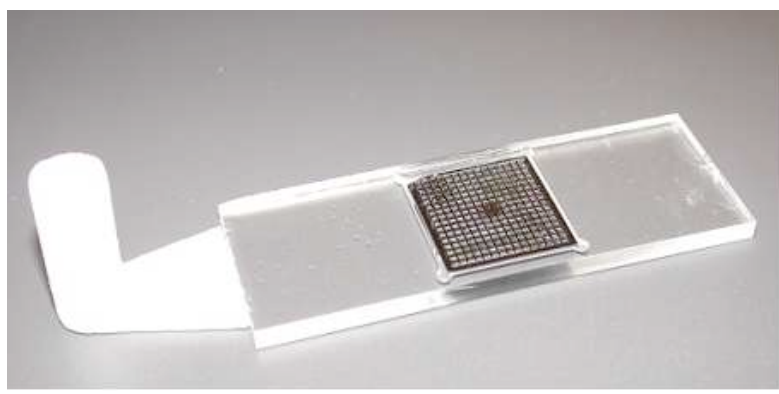

(b)

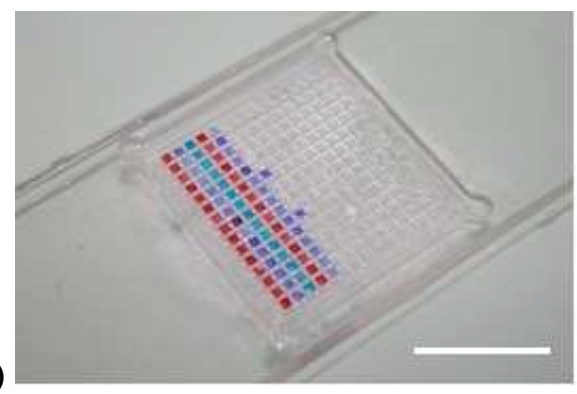

Figure 2: Photo of the composite master part used for the biochip fabrication (a); photo of the polymer duplicated biochip, obtained through vacuum casting: colored liquid are spotted on the chip (b) (scale bar is $1 \mathrm{~cm}$ ). 


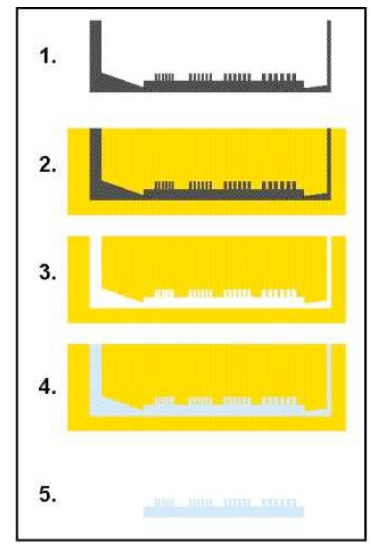

Figure 3: Schematic illustration of the vacuum casting process

The master part, shown in figure 2 , is a composite object whose sub-units are made through various technologies. In our case three subunits are assembled 1) one subunit being an acrylic plate which has been conventionally machined, 2) a subunit made through fused deposition modeling technology and 3) a subunit made of silicon being etched through MEMS technology. The opportunity of making composite master part allows to compose with best available fabrication technologies according to requirements in term of surface roughness, aspect-ratio and desired shape. The dimensions of the biochip fitted the range of the vacuum casting technique. The master part was successfully duplicated up to 40 times with one silicone flexible mould. The figure shows pictures of the original and one duplicated part. The schematic illustration of the vacuum casting fabrication process is shown in Figure. 3. Vent and sprue bushing channels are added to a composite master part fabricated through standard micro-fabrication technologies (step 1). Various materials are suitable and can be associated to form the master part. A silicone prepolymer is mixed with hardener, degassed and then poured around the master part to realize a female flexible mould (step 2). The master part is withdrawn from the silicone mould (step 3). Polymer liquid resin (MCP6091) is then cast under vacuum in the empty flexible female mould (step 4). The polymer resin is baked for 3 hours at $70^{\circ} \mathrm{C}$. The hardened polymer part is then withdrawn from the mould (step 5). It is a transparent and accurate duplication of the master part made with a chosen polymer.

\section{Biological application : transfection experiment}

In this section we present the transfection experiment on cells cultured on the biochip which microfabrication is described in the above paragraph. Biocompatibility tests which have been performed on the biochip are then presented. Finally, cell loading, transfection and culture on the biochip is described.

\subsection{Biocompatibility}

The biocompatibility of the polymer material used was monitored by cell proliferation experiments in the polymer micro-wells over one week. Good viability of cells on the chip was observed with the chosen polymer (see figure 4). The material showed to be suitable for fluorescence observation. 


\subsection{Transfection experiment}

\subsubsection{Cellchip printing}

The feasibility of parallel transfection with different transgene on the plastic biochip, without crosscontamination between cells cultured in adjacent micro-chambers was demonstrated. For that purpose, plasmids expressing two different fluorescent proteins (eGFP and DsRed2) were alternatively used for the transfection.

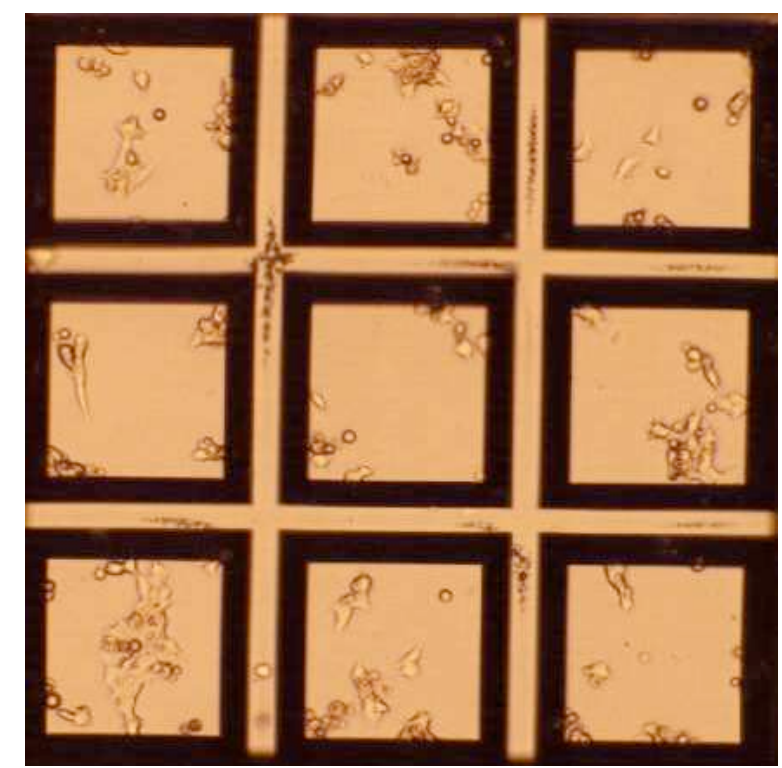

Figure 4: Pictures of FAO cells on the biochip after 72 hours of culture. The cells stretch at the bottom of the micro-wells.

The general lipidic reverse-transfection procedure used here was derived from previous work [31] except for the absence of gelatin. Briefly, 1 microgram of each plasmid - either pEGFP-C1 or pDsRed2 (Clontech, Paolo Alto, CA) encoding respectively a green and a red fluorescence protein, were diluted with $13.2 \mu 1$ of EC buffer (Effectene kit, Qiagen). Four microliters of Enhancer supplemented with $2.4 \mu \mathrm{l}$ of a $1.5 \mathrm{M}$ sucrose solution and $4 \mu \mathrm{l}$ of Effectene reagent were successively added to the mixture.

After 10 minutes at room temperature, the solution was transferred into a 96-wells plate for microarray printing. The microfabricated chip were printed at the bottom of each microwell at room temperature with a Microgrid II Biorobotics (Cambridge, UK) arrayer equipped with pin (usually used for DNA microarrays manufacturing). The spots were about $100 \mu \mathrm{m}$ in diameter as verified by printing of a blue colored solution (see Figure 5). DsRed2 and eGFP encoding plasmids were alternatively arrayed on adjacent rows of the chip. After printing, the chip was dried and stored in a dessicator for subsequent use.3.2.2. Cell culture and Reverse Transfection

Human Embryonic Kidney cell line HEK293T were grown at $37^{\circ} \mathrm{C}$ in a $5 \% \mathrm{CO} 2$ humidified atmosphere in Dulbecco's modified Eagle's medium containing $4.5 \mathrm{~g} / \mathrm{L}$ glucose supplemented with 
$10 \%$ FCS, 100,000U/L penicillin, $50 \mathrm{mg} / \mathrm{L}$ streptomycin and $200 \mathrm{mM}$ glutamine. Cells were seeded onto the chip (450,000 cells in $1 \mathrm{~mL}$ of medium) and cultured in $100 \mathrm{~mm}$ Petri dishes containing PBS, and cultured for $24-72 \mathrm{hrs}$ at $37^{\circ} \mathrm{C} / 5 \% \mathrm{CO} 2$.

\subsubsection{Transfection analysis}

Transfection was assessed by fluorescence measurement on a fluorescence microscope and array images were captured with a Zeiss Axiovert microscope with fluorescent light emission systems for EGFP (Ex $488 \mathrm{~nm}$, Em $520 \mathrm{~nm}$ ) and DsRed2 (Ex $558 \mathrm{~nm}$, Em $583 \mathrm{~nm}$ ) detection and a digital camera for image acquisition.

\subsubsection{Results:}

72 hours after transfection, phenotypical effects of gene overexpression could be assessed on the array by visualization of green (EGFP) and red (DsRed2) fluorescence using a fluorescence microscope. Successful expression of both genes without cross contamination could be observed as shown in Figure 5. The polymer proved to be compatible with cell transfection, and the microchip format scalable for highly parallel transfection.

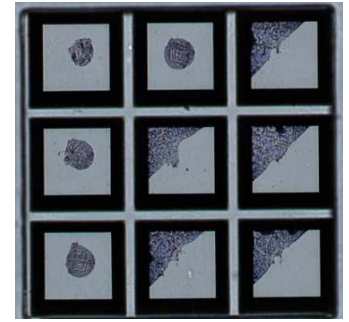

a.

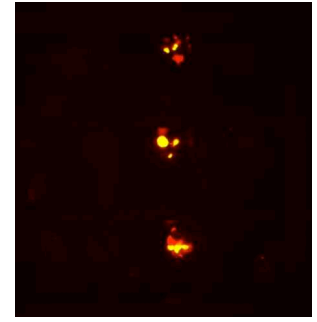

b.

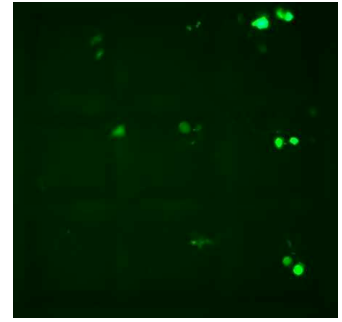

d.

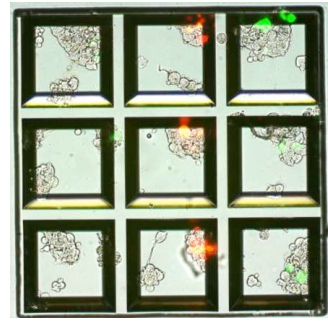

d.

Figure 5 : Lipidic reverse-transfection experiment on HEK 293T cells on the biochip.

(a) Visualization of the spotting with a blue colored solution. (b) and (c) Two plasmids expressing different fluorescent proteins (DsRed2 and eGFP) were spotted in adjacent wells onto the array and cells were seeded. Red (b) and green (c) fluorescence corresponding to DsRed2 and eGFP expression was assessed using a microscope. (d) Successful expression of both genes in HEK293T cells without cross contamination between adjacent wells could be observed after $72 \mathrm{H}$ culture.

\section{Conclusion}

A new micro-fabrication technology was presented in this paper, which combines the advantages of micro-size features realization capabilities, while optimizing cost reduction when small series market niches are targeted. This new technology was successfully evaluated through the microfabrication of a transparent polymer biochip for cell transfection, and showed satisfactory biological and fluidic characteristics. Transfection of HEK293T cells cultured on the chip was achieved, using two plasmids encoding fluorescent proteins. Successful expression of both genes without cross contamination 
between microwells could be observed allowing to envision highly parallel and differential transfection on that format.

In the present post-sequencing era, there is an actual need for new tools allowing large scale gene function studies. Cell microarrays allowing transfection of thousands of nucleic acids in parallel and the subsequent analysis of the phenotypic consequences of such perturbations are indeed very promising in that field. They allow to increase the throughput of the studies and to reduce the amount of material needed. The format described here present several key features: a high number of wells, a low amount of nucleic acid needed for transfection, along with a low manufacturing cost and imaging analysis compatibility (transparency, microscope slide format...).

This new micro-fabrication technology of polymer biochips is advantageous for 1) its high accuracy in the replication 2) the uniform retractation which was observed, remaining under 1\%,3) the low temperature process which is used, rendering electrical or optical part integration highly possible. Moreover, as shown on Figure 6, this vacuum casting of plastic micro-sized chip may be cost competitive, between huge polymer production techniques and hand-made micro-fabrication technologies.

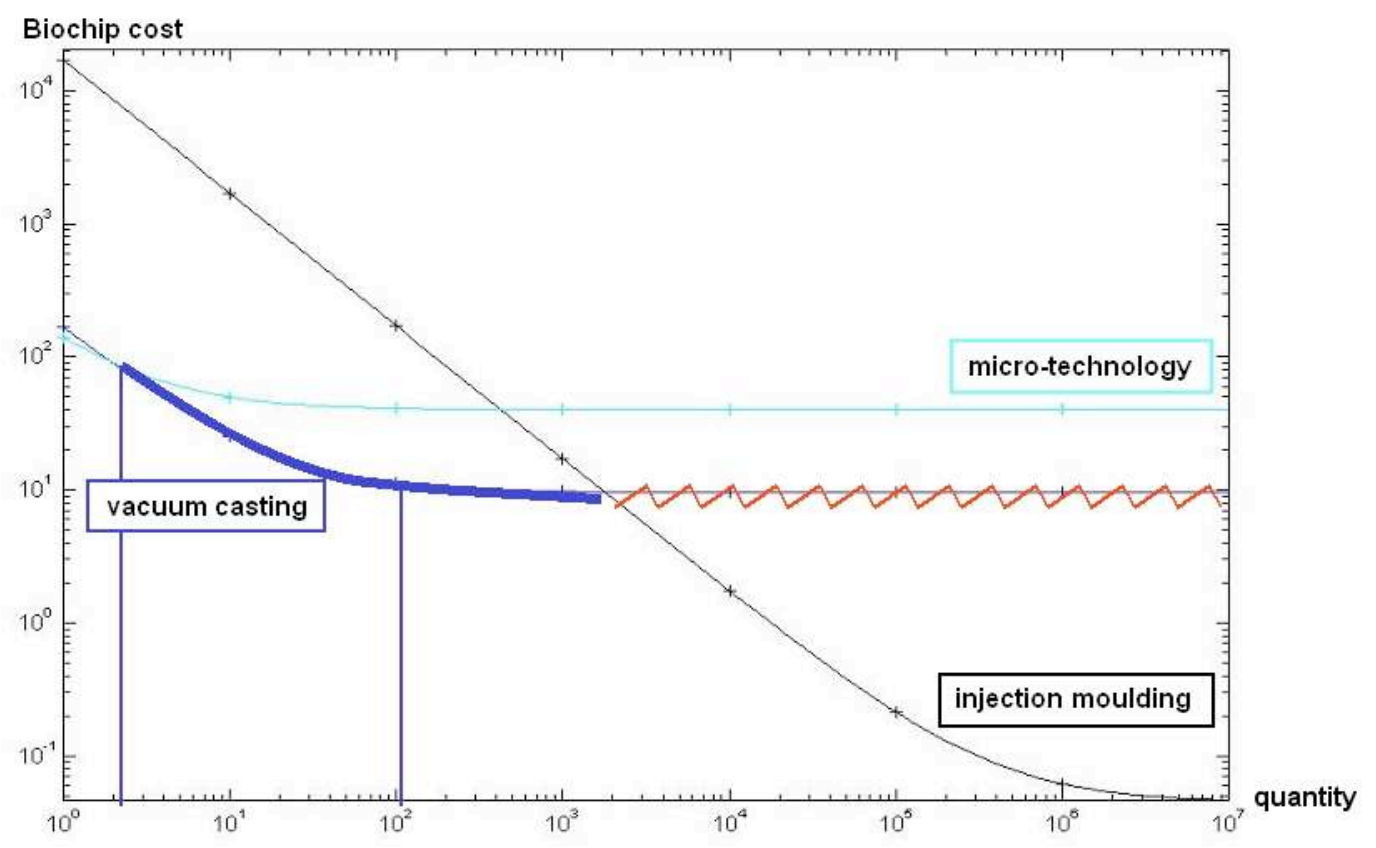

Figure 6 : Biochip microarray cost versus production volume compared for injection molding, microfabrication technology and vacuum-casting process.

\section{References}

[1] Seger U, Gavad S, Johann R, Bertsch A and Renaud P 2004 Lab on a Chip 4 2-148

[2] T. Braschker, R. Johann, M. Heule, L. Metret and P. Renaud 2005 Lab on a Chip. 5 5-553

[3] Huang Y and Rubinsky B 2001 Sensors and Actuators A 89242 
[4] Khine M, Lau A, Ionescu-Zanetti C, Jeonggi S and Lee L P 2005 Lab on a Chip 538

[5] Seo J, Ionescu-Zanetti C, Diamond J, Lal R and Lee L P 2004 Appl. Phys. Letters 841973

[6] Raiteri R, Grattarola M and Berger R 2002 Materials Today. 5 1-22

[7] Kuschel M, Buhlmann C and Preckel T 2005 J. Association for Laboratory Automation 10 5-319

[8] Csako G 2006 Clinica Chimica Acta 2636

[9] Bailey S N, Randy Z and Sabatini D M 2002 Drug Discovery Today 7 18-113

[10] Ringerike T, Ulleras E, Nilsson G, Vandebriel R, van Loveren H, Dastych J and Lovik M 2003

Toxicology Letters 144 1-34

[11] Freitag A, Vogel D, Scholz R and Dietrich T R 2001 J. Association for Laboratory Automation $64-45$

[12] Cheng Y, Sugioka K and Midorikawa K 2005 Appl. Surface Science 248172

[13] Lee K B and Lin L 2004 Sensors and Actuators A 11144

[14] Rodriguez I, Spicar-Mihalic P, Kuyper C L, Fiorini G S and Chiu D T 2003 Analytica Chimica Acta 496205

[15] Lee H G 1997 J. Chromatography A 790215

[16] Sammoura F, Su Y S, Cai Y, Chi C Y, Elamaran B, Lin L and Chiao J C 2005 Sensors and Actuators A (in press)

[17] Goretty Alonso-Amigo A 2000 J. Association for Laboratory Automation 5 6-96

[18] Duffy D, Mac Donald C, Schueller O and Whitesides G M 1998 Anal. Chem. 704974

[19] Gale M T, Gimkiewicz C, Obi S, Schnieper M, Sochtig J, Thiele H and Westenhofer S 2005 Optics and Lasers in Eng. 43373

[20] Kim J T and Choi C G 2006 Optics Comm. 25772

[21] Lee G B, Chen S H, Huang G R, Sung W C and Lin Y H 2001 Sensors and Actuators B 75142

[22] Lin L, Cheng T and Chiu C J 1998 Microsystem Technologies 4113

[23] Shen X J, Pan L W and Lin L 2002 Sensors and Actuators A 97-98 428

[24] Becker H and Heim U 2000 Sensors and Actuators A 83130

[25] Becker H and Locascio L E 2002 Talanta 56267

[26] Su Y C, Shah J and Lin L 2004 J. Micromech. Microeng. 14415

[27] Shen Y K and Wu W Y 2002 Int. Comm. Heat Mass Transfer 29 3-423

[28] Park Y, Cho H, Mok Son Y, Park J Y, Pu L, Yoon S K, Hong Y S and Lee H K 2005 Microelect. Eng. 82 1-11

[29] Marquette C A and Blum L J 2004 Analytica Chimica Acta 506127

[30] Denoual M, Mognol P and LePioufle B 2005 J. Eng. Manufacture Part B 2199

[31] Baghdoyan S, Roupioz Y, Pitaval A, Castel D, Khomyakova E, Papine A, Soussaline F and Gidrol X 2004 Nucleic Acids Res. 32 e77 